Acta Crystallographica Section E

Structure Reports

Online

ISSN 1600-5368

\section{Hümeyra Batı, ${ }^{a}$ Ayșin Zülfikaroğlu, a Murat Taș, ${ }^{a}$ Omer Andac ${ }^{a}$ and William T. A. Harrison ${ }^{b}$ *}

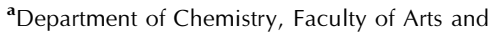
Sciences, Ondokuz Mayıs University, 55139 Kurupelit Samsun, Turkey, and ${ }^{\mathbf{b}}$ Department of Chemistry, University of Aberdeen, Meston Walk, Aberdeen AB24 3UE, Scotland

Correspondence e-mail: oandac@omu.edu.tr

\section{Key indicators}

Single-crystal X-ray study

$T=293 \mathrm{~K}$

Mean $\sigma(\mathrm{C}-\mathrm{C})=0.002 \AA$

$R$ factor $=0.034$

$w R$ factor $=0.093$

Data-to-parameter ratio $=26.3$

For details of how these key indicators were automatically derived from the article, see http://journals.iucr.org/e.

\title{
Bis[1-(2,6-dimethylanilino)propane-1,2-dione dioximato]nickel(II)
}

The structure of the title complex consists of isolated $\left[\mathrm{Ni}\left(\mathrm{C}_{11} \mathrm{H}_{14} \mathrm{~N}_{3} \mathrm{O}_{2}\right)_{2}\right]$ units. The $\mathrm{Ni}$ atom is coordinated by four oxime $\mathrm{N}$ atoms in distorted square-planar geometry and lies on an inversion centre. The structure is stabilized by strong intramolecular $\mathrm{N}-\mathrm{H} \cdots \mathrm{O}$ and $\mathrm{O}-\mathrm{H} \cdots \mathrm{O}$ hydrogen bonds and a possible $\mathrm{N}-\mathrm{H} \cdots \pi$ intermolecular interaction.

\section{Comment}

This work is part of our ongoing research on the synthesis and characterization of new vic-dioximes and their transition metal complexes (Zülfikaroglu et al., 2003). Metal complexes of various glyoximate ligands have long been of importance in analytical chemistry and medicine (Chakravorty, 1974; Michael et al., 2000).

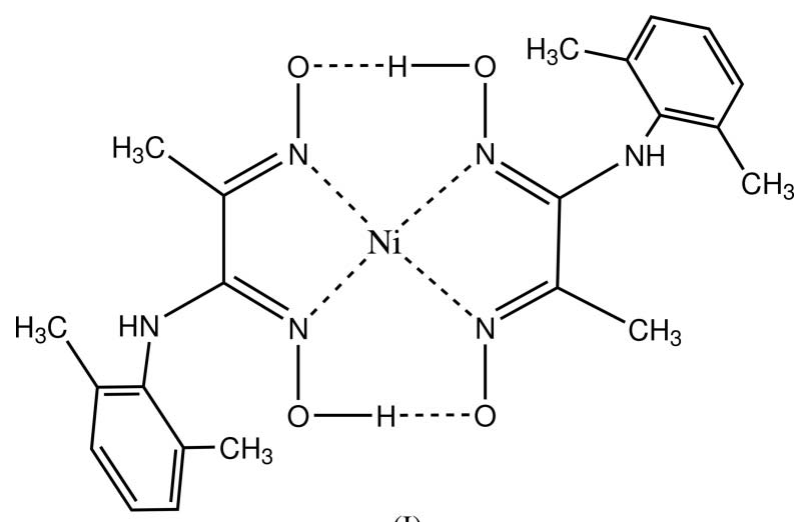

(I)

In the title compound, (I), alternately named bis[N-(2,6dimethylphenyl)aminomethylglyoximato- $N, N^{\prime}$ ]nickel(II) (Fig. 1), the Ni atom, which lies on a site of $\overline{1}$ symmetry, is coordinated by four oxime $\mathrm{N}$ atoms arising from two bidentate ligand molecules. The local coordination of the $\mathrm{NiN}_{4}$ chromophore is distorted square planar ( $D_{2 h}$ symmetry). The $\mathrm{Ni}-\mathrm{N}$ and $\mathrm{O} 1 \cdots \mathrm{O} 2^{\mathrm{i}}$ distances (Tables 1 and 2; symmetry code as in these tables) are similar to the distances found in the related complexes bis[ $N$-(2,6-dimethylphenyl)aminoglyoximato- $\left.N, N^{\prime}\right]$ nickel(II) (Ülkü et al., 1996), bis[N-(4methylphenyl)aminoglyoximato- $\left.N, N^{\prime}\right]$ nickel(II) (Isik et al., 2000), bis[ $N$-(2,6-dimethylphenyl)aminophenylglyoximato$\left.\kappa^{2} N, N^{\prime}\right]$ nickel(II) dimethyl sulfoxide solvate (Batı et al., 2004) and bis[ $N$-(4-methoxyphenyl)aminomethylglyoximato]nickel(II) (Bat1 et al., 2005). In these, one $\mathrm{Ni}-\mathrm{N}$ bond is significantly longer than the other (by between 0.02 and $0.05 \AA)$. This difference can possibly be attributed to the different groups attached to oxime atoms C9 and C10.
Received 8 September 2005 Accepted 13 September 2005 Online 17 September 2005
(C) 2005 International Union of Crystallography Printed in Great Britain - all rights reserved 
The different $\mathrm{N}-\mathrm{O}$ bond lengths reflect the chemically distinct $\mathrm{O}$ atoms. The oxime group has an $E$ configuration with planar $\mathrm{O} 1-\mathrm{N} 2-\mathrm{C} 9-\mathrm{C} 10$. The oxime $\mathrm{OH}$ group is adjacent to the bridging amine group in all complexes, and in (I) accepts an intraligand $\mathrm{N}-\mathrm{H} \cdots \mathrm{O}$ bond. The benzene and fivemembered chelate $\left(\mathrm{NiC}_{2} \mathrm{~N}_{2}\right)$ rings in (I) are essentially planar, with r.m.s. deviations of only 0.0045 and $0.0159 \AA$.

Comparision of the bond lengths of the oxime group with those of the free ligand (Hökelek et al., 2001) reveals that, upon complex formation, the $\mathrm{N} 2-\mathrm{O} 1, \mathrm{~N} 3-\mathrm{O} 2$ and $\mathrm{C} 9-\mathrm{C} 10$ distances are shortened by $0.040,0.078$ and $0.018 \AA$, respectively, whereas the $\mathrm{C} 9-\mathrm{N} 2$ and $\mathrm{C} 10-\mathrm{N} 3$ distances are increased by 0.013 and $0.028 \AA$, respectively.

The intramolecular inter-ligand $\mathrm{O} \cdots \mathrm{O}$ separations in these compounds are all similar, lying between 2.462 (3) and 2.547 (3) $\AA$. Such short $\mathrm{O} \ldots \mathrm{O}$ separations are often associated with symmetrical $\mathrm{O} \cdots \mathrm{H} \cdots \mathrm{O}$ hydrogen bonds (Chakravorty, 1974). In (I), one of the O-bound acidic $\mathrm{H}$ atoms is lost from each ligand during complex formation and the remaining O-bound $\mathrm{H}$ atom participates in a very strong intramolecular hydrogen bond to the adjacent $\mathrm{O}$ atom (Table 2). The $\mathrm{H}$ atom was clearly visible in a difference map and, like the other complexes noted above, the $\mathrm{O}-\mathrm{H} \cdots \mathrm{O}$ bond is not symmetrical.

An analysis of the intermolecular contacts in (I) with PLATON (Spek, 2003) revealed a possible weak $\mathrm{N}-$ $\mathrm{H} \cdots \pi(-x, 1-y, 2-z)$ interaction between the amine $\mathrm{H}$ atom and an adjacent benzene ring (atoms $\mathrm{C} 1-\mathrm{C} 6)$ with an $\mathrm{H} \cdots \pi$ distance of 2.965 (16) $\AA$.

\section{Experimental}

1-(2,6-Dimethylphenylamino)propane-1,2-dione dioxime $(L)$ was prepared according to the method of Hökelek et al. (2001). A solution of $\mathrm{NiCl}_{2} \cdot 6 \mathrm{H}_{2} \mathrm{O}(0.48 \mathrm{~g} .2 \mathrm{mmol})$ in ethanol-water (1:1) was added dropwise to a solution of $L(0.882 \mathrm{~g} .4 \mathrm{mmol})$ in ethanol $(20 \mathrm{ml})$. A $1 \%$ solution of $\mathrm{KOH}$ in water was then dripped slowly into the mixture until the $\mathrm{pH}$ reached 5.5. The resulting precipitate was removed by suction filtration, washed and dried in vacuo. Recrystallization from a chloroform-ethanol mixture (2:1) gave orange rod crystals of (I).

$$
\begin{aligned}
& \text { Crystal data } \\
& {\left[\mathrm{Ni}\left(\mathrm{C}_{11} \mathrm{H}_{14} \mathrm{~N}_{3} \mathrm{O}_{2}\right)_{2}\right]} \\
& M_{r}=499.21 \\
& \text { Monoclinic, } P 2_{1} / c \\
& a=8.1081(4) \AA \\
& b=16.0311(8) \AA \\
& c=8.9223(4) \AA \\
& \beta=94.202(1)^{\circ} \\
& V=1156.62(10) \AA^{3} \\
& Z=2
\end{aligned}
$$$$
\begin{aligned}
& D_{x}=1.433 \mathrm{Mg} \mathrm{m}^{-3} \\
& \text { Mo } K \alpha \text { radiation } \\
& \text { Cell parameters from } 4650 \\
& \quad \text { reflections } \\
& \theta=2.5-32.0^{\circ} \\
& \mu=0.88 \mathrm{~mm}^{-1} \\
& T=293(2) \mathrm{K} \\
& \text { Rod, orange } \\
& 0.49 \times 0.30 \times 0.24 \mathrm{~mm}
\end{aligned}
$$

Data collection

Bruker SMART 1000 CCD areadetector diffractometer $\omega$ scans

Absorption correction: multi-scan (SADABS; Sheldrick, 1997)

$T_{\min }=0.736, T_{\max }=0.810$

11778 measured reflections

independent reflections 2988 reflections with $I>2 \sigma(I)$

$R_{\text {int }}=0.021$

$\theta_{\max }=32.5^{\circ}$

$h=-12 \rightarrow 11$

$k=-24 \rightarrow 16$

$l=-13 \rightarrow 13$

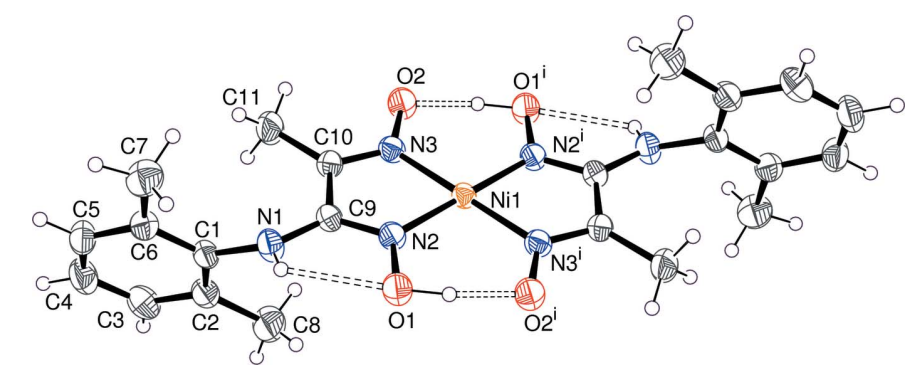

Figure 1

View of (I) showing $40 \%$ probability displacement ellipsoids (arbitrary spheres for the $\mathrm{H}$ atoms) and hydrogen bonds as dashed lines. [Symmetry code: (i) $1-x, 1-y, 1-z$.]

\section{Refinement}

Refinement on $F^{2}$

$R\left[F^{2}>2 \sigma\left(F^{2}\right)\right]=0.034$

$w R\left(F^{2}\right)=0.093$

$S=0.98$

4184 reflections

159 parameters

$\mathrm{H}$ atoms treated by a mixture of independent and constrained refinement

$w=1 /\left[\sigma^{2}\left(F_{\mathrm{o}}^{2}\right)+(0.0541 P)^{2}\right]$

where $P=\left(F_{\mathrm{o}}{ }^{2}+2 F_{\mathrm{c}}{ }^{2}\right) / 3$

$(\Delta / \sigma)_{\max }<0.001$

$\Delta \rho_{\max }=0.45 \mathrm{e}^{-3}$

$\Delta \rho_{\min }=-0.16 \mathrm{e}^{-3}$

Table 1

Selected geometric parameters $\left(\AA{ }^{\circ}\right)$.

\begin{tabular}{lrlr}
\hline $\mathrm{Ni} 1-\mathrm{N} 2$ & $1.8397(11)$ & $\mathrm{Ni} 1-\mathrm{N} 3$ & $1.8779(11)$ \\
$\mathrm{N} 2-\mathrm{Ni1}-\mathrm{N} 3^{\mathrm{i}}$ & $97.62(5)$ & $\mathrm{N} 2-\mathrm{Ni1}-\mathrm{N} 3$ & $82.38(5)$ \\
\hline
\end{tabular}

Symmetry code: (i) $1-x, 1-y, 1-z$.

Table 2

Hydrogen-bond geometry $\left(\AA{ }^{\circ}\right)$.

\begin{tabular}{lllll}
\hline$D-\mathrm{H} \cdots A$ & $D-\mathrm{H}$ & $\mathrm{H} \cdots A$ & $D \cdots A$ & $D-\mathrm{H} \cdots A$ \\
\hline $\mathrm{O} 1-\mathrm{H} 1 O \cdots \mathrm{O} 2^{\mathrm{i}}$ & $0.86(1)$ & $1.65(1)$ & $2.4972(14)$ & $171(2)$ \\
$\mathrm{N} 1-\mathrm{H} 1 N \cdots \mathrm{O} 1$ & $0.82(1)$ & $2.20(2)$ & $2.6361(16)$ & $113(1)$ \\
\hline
\end{tabular}

Symmetry code: (i) $1-x, 1-y, 1-z$.

The $\mathrm{O}$ - and $\mathrm{N}$-bound $\mathrm{H}$ atoms were found in difference maps and were refined with distance restraints $[\mathrm{O}-\mathrm{H}=0.84$ (2) $\AA$ and $\mathrm{N}-\mathrm{H}=$ $0.86(2) \AA]$ and with $U_{\text {iso }}(\mathrm{H})=1.2 U_{\text {eq }}$ (carrier). C-bound $\mathrm{H}$ atoms were placed in calculated positions $(\mathrm{C}-\mathrm{H}=0.93-0.96 \AA)$ and refined as riding, with $U_{\text {iso }}(\mathrm{H})=1.2 U_{\text {eq }}($ carrier $)$ or $U_{\text {iso }}(\mathrm{H})=1.5 U_{\text {eq }}($ methyl carrier). The $-\mathrm{CH}_{3}$ groups were rotated to fit the electron density.

Data collection: SMART (Bruker, 1999); cell refinement: SAINT (Bruker, 1999); data reduction: $S A I N T$; $\operatorname{program}(\mathrm{s})$ used to solve structure: SHELXS97 (Sheldrick, 1997); program(s) used to refine structure: SHELXL97 (Sheldrick, 1997); molecular graphics: ORTEP-3 (Farrugia, 1997); software used to prepare material for publication: SHELXL97.

The authors thank Ondokuz Mayıs University for financial support.

\section{References}

Batı, H., Daĝ, C., Soylu, M. S., Taş, M., Çalışkan, N. \& Büyükgüngör, O. (2005). Acta Cryst. E61, m1866-m1868. 


\section{metal-organic papers}

Batı, H., Zülfikaroglu, A., Taş, M., Çalışkan, N., Soylu, S., Andac, O. \& Büyükgüngör, O. (2004). Acta Cryst. E60, m1334-m1336.

Bruker (1999). SMART and SAINT. Bruker AXS Inc., Madison, Wisconsin, USA.

Chakravorty, A. (1974). Coord. Chem. Rev. 13, 1-46.

Farrugia, L. J. (1997). J. Appl. Cryst. 30, 565.

Hökelek, T., Zülfikaroglu, A. \& Batı, H. (2001). Acta Cryst. E57, o1247-o1249.

Isik, S., Ozturk, S., Erdonmez, A., Macit, M. \& Fun, H.-K. (2000). Anal. Sci. 16, 559-560.
Michael, J. P., Anthony, W. A. \& Raymond, J. B. (2000). Inorg. Chim. Acta, 300-302, 992-1003.

Sheldrick, G. M. (1997). SADABS, SHELXS97 and SHELXL97. University of Göttingen, Germany.

Spek, A. L. (2003). J. Appl. Cryst. 36, 7-13.

Ülkü, D., Ercan, F., Macit, M. \& Gulce, A. (1996). Acta Cryst. C52, 26802682.

Zülfikaroglu, A., Taş, M., Batı, H. \& Batı, B. (2003). Synth. React. Inorg. Met.Org. Chem. 33, 625-638. 


\section{supporting information}

Acta Cryst. (2005). E61, m2033-m2035 [doi:10.1107/S1600536805028965]

\section{Bis[1-(2,6-dimethylanilino) propane-1,2-dione dioximato]nickel(II) \\ Hümeyra Batı, Ayşin Zülfikaroğlu, Murat Taş, Omer Andac and William T. A. Harrison}

\section{S1. Comment}

This work is part of our ongoing research on the synthesis and characterization of new vic dioximes and their transition metal complexes (Zülfikaroglu et al., 2003). Metal complexes of various glyoximate ligands have long been of importance in analytical chemistry and medicine (Chakravorty, 1974; Michael et al., 2000). In the title compound, (I), alternately named bis[N-(2,6-dimethylphenyl)aminomethylglyoximato- $\left.N, N^{\prime}\right]$ nickel(II) (Fig. 1), the $\mathrm{Ni}$ atom that lies on a site of -1 symmetry, is coordinated by four oxime $\mathrm{N}$ atoms arising from two bidentate ligand molecules. The local coordination of the $\mathrm{NiN}_{4}$ chromophore is distorted square planar $\left(D_{2 h}\right.$ symmetry). The $\mathrm{Ni}-\mathrm{N}$ and $\mathrm{O} 1 \cdots \mathrm{O} 2^{\mathrm{i}}$ distances (Tables 1 and 2) are similar to the distances found in the related complexes bis[ $N$-(2,6-dimethylphenyl)aminoglyoximato$\left.N, N^{\prime}\right]$ nickel(II) (Ulku et al., 1996), bis[N-(4-methylphenyl) aminoglyoximato- $\left.N, N^{\prime}\right]$ nickel(II) (Isik et al., 2000), bis[ $N$-(2,6-dimethylphenyl)aminophenylglyoximato- $\left.\kappa^{2} N, N^{\prime}\right]$ nickel(II) dimethyl sulfoxide solvate (Bat1 et al., 2004) and bis[N-(4-methoxyphenyl)aminomethylglyoximato]nickel(II) (Batı et al., 2005). In these, one $\mathrm{Ni}-\mathrm{N}$ bond is significantly longer than the other (by between 0.02 and $0.05 \AA$ ). This difference can possibly be attributed to the different groups attached to oxime atoms $\mathrm{C} 9$ and $\mathrm{C} 10$. The intramolecular, inter-ligand, $\mathrm{O} \cdots \mathrm{O}$ separations in these compounds are all similar, lying between 2.462 (3) and 2.547 (3) $\AA$. Such short O $\cdots \mathrm{O}$ separations are often associated with symmetrical $\mathrm{O} \cdots \mathrm{H} \cdots \mathrm{O}$ hydrogen bonds (Chakravorty, 1974). In (I), one of the O-bound acidic $\mathrm{H}$ atoms is lost from each ligand during complex formation and the remaining O-bound $\mathrm{H}$ atom participates in a very strong intramolecular hydrogen bond to the adjacent $\mathrm{O}$ atom (Table 2). The $\mathrm{H}$ atom was clearly visible in a difference map and, like the other complexes noted above, the $\mathrm{O}-\mathrm{H} \cdots \mathrm{O}$ bond is not symmetrical. The different $\mathrm{N}-\mathrm{O}$ bond lengths reflect the chemically distinct $\mathrm{O}$ atoms. The oxime group has an $E$ configuration with planar $\mathrm{O} 1-\mathrm{N} 2-\mathrm{C} 9-\mathrm{C} 10$. The oxime $-\mathrm{OH}$ group is adjacent to the bridging amine group in all complexes, and in (I) accepts an intraligand $\mathrm{N}-\mathrm{H} \cdots \mathrm{O}$ bond. The phenyl and five-membered chelate $\left(\mathrm{NiC}_{2} \mathrm{~N}_{2}\right)$ rings in (I) are almost planar, with r.m.s deviations of 0.0045 and $0.0159 \AA$ only.

Comparision of the bond lengths of the oxime group with those of the free ligand (Hökelek et al., 2001) reveals that, upon complex formation, the $\mathrm{N} 2-\mathrm{O} 1, \mathrm{~N} 3-\mathrm{O} 3$ and $\mathrm{C} 9-\mathrm{C} 10$ distances are shortened by $0.040,0.078$ and $0.018 \AA$, whereas the $\mathrm{C} 9-\mathrm{N} 2$ and $\mathrm{C} 10-\mathrm{N} 3$ distances are increased by 0.013 and $0.028 \AA$.

An analysis of the inter-molecular contacts in (I) with PLATON (Spek, 2003) reveals a possible weak $\mathrm{N}-\mathrm{H} \cdots \pi(-x, 1-$ $y, 2-z$ ) interaction between the amine $\mathrm{H}$ atom and an adjacent phenyl ring (atoms $\mathrm{C} 1-\mathrm{C} 6$ ) with an $\mathrm{H} \cdots \pi$ distance of $2.965(16) \AA$.

\section{S2. Experimental}

1-(2,6-Dimethylphenylamino)propane-1,2-dione dioxime $(L)$ was prepared according to the method of Hökelek et al. (2001). A solution of $\mathrm{NiCl}_{2} \cdot 6 \mathrm{H}_{2} \mathrm{O}(0.48 \mathrm{~g}$. $2 \mathrm{mmol})$ in ethanol-water $(1: 1)$ was added dropwise to a solution of $L(0.882 \mathrm{~g}$. $4 \mathrm{mmol}$ ) in ethanol $(20 \mathrm{ml})$. Then, a $1 \%$ solution of $\mathrm{KOH}$ in water was dripped slowly into the mixture until the $\mathrm{pH}$ reached 5.5. The resulting precipitate was removed by suction filtration, washed and dried in vacuo. Recrystallization 
from a chloroform-ethanol mixture (2:1) gave orange rod crystals of (I).

\section{S3. Refinement}

The $\mathrm{O}-$ and $\mathrm{N}$-bound $\mathrm{H}$ atoms were found in difference maps and were refined with distance restraints $[\mathrm{O}-\mathrm{H}=0.84(2)$ $\AA$ and $\mathrm{N}-\mathrm{H}=0.86(2) \AA]$ and with $U_{\text {iso }}(\mathrm{H})=1.2 U_{\text {eq }}$ (carrier) to yield the final values given in Table 2. C-bound $\mathrm{H}$ atoms were placed in calculated positions $(\mathrm{C}-\mathrm{H}=0.93-0.96 \AA)$ and refined as riding with $U_{\text {iso }}(\mathrm{H})=1.2 U_{\text {eq }}($ carrier $)$ or $U_{\text {iso }}(\mathrm{H})$ $=1.5 U_{\mathrm{eq}}$ (methyl carrier). The $-\mathrm{CH}_{3}$ groups were rotated to fit the electron density.

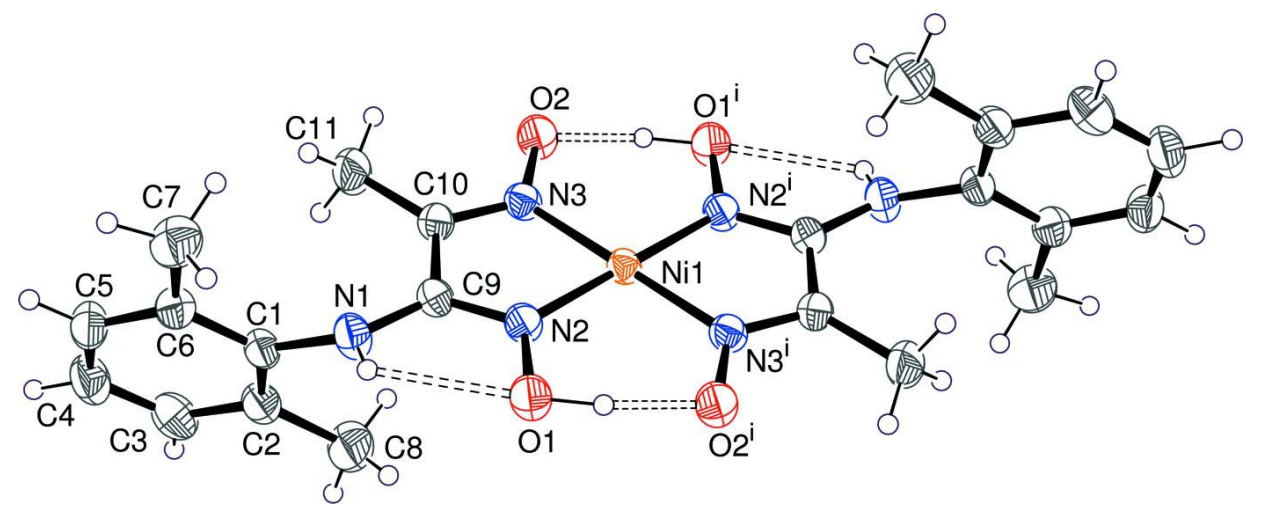

\section{Figure 1}

View of (I) showing $40 \%$ probability displacement ellipsoids (arbitrary spheres for the $\mathrm{H}$ atoms) and hydrogen bonds as dashed lines. [Symmetry code: (i) $1-x, 1-y, 1-z$.]

\section{Bis[1-(2,6-dimethylanilino)propane-1,2-dione dioximato]nickel(II)}

\section{Crystal data}

$\left[\mathrm{Ni}\left(\mathrm{C}_{11} \mathrm{H}_{14} \mathrm{~N}_{3} \mathrm{O}_{2}\right)_{2}\right]$

$M_{r}=499.21$

Monoclinic, $P 2_{1} / c$

Hall symbol: $-\mathrm{P} 2 \mathrm{ybc}$

$a=8.1081(4) \AA$

$b=16.0311(8) \AA$

$c=8.9223(4) \AA$

$\beta=94.202(1)^{\circ}$

$V=1156.62(10) \AA^{3}$

$Z=2$

\section{Data collection}

Bruker SMART 1000 CCD area-detector diffractometer

Radiation source: fine-focus sealed tube

Graphite monochromator

$\omega$ scans

Absorption correction: multi-scan

(SADABS; Sheldrick, 1997)

$T_{\min }=0.736, T_{\max }=0.810$

\section{Refinement}

Refinement on $F^{2}$

Least-squares matrix: full

$R\left[F^{2}>2 \sigma\left(F^{2}\right)\right]=0.034$
$F(000)=524$

$D_{\mathrm{x}}=1.433 \mathrm{Mg} \mathrm{m}^{-3}$

Mo $K \alpha$ radiation, $\lambda=0.71073 \AA$

Cell parameters from 4650 reflections

$\theta=2.5-32.0^{\circ}$

$\mu=0.88 \mathrm{~mm}^{-1}$

$T=293 \mathrm{~K}$

Rod, orange

$0.49 \times 0.30 \times 0.24 \mathrm{~mm}$

11778 measured reflections

4184 independent reflections

2988 reflections with $I>2 \sigma(I)$

$R_{\text {int }}=0.021$

$\theta_{\text {max }}=32.5^{\circ}, \theta_{\text {min }}=2.5^{\circ}$

$h=-12 \rightarrow 11$

$k=-24 \rightarrow 16$

$l=-13 \rightarrow 13$

$w R\left(F^{2}\right)=0.093$

$S=0.98$

4184 reflections 
159 parameters

0 restraints

Primary atom site location: structure-invariant direct methods

Secondary atom site location: none

Hydrogen site location: difmap (O-H and N-H)

and geom $(\mathrm{C}-\mathrm{H})$
$\mathrm{H}$ atoms treated by a mixture of independent and constrained refinement

$w=1 /\left[\sigma^{2}\left(F_{\mathrm{o}}^{2}\right)+(0.0541 P)^{2}\right]$

where $P=\left(F_{\mathrm{o}}^{2}+2 F_{\mathrm{c}}^{2}\right) / 3$

$(\Delta / \sigma)_{\max }<0.001$

$\Delta \rho_{\max }=0.45$ e $\AA^{-3}$

$\Delta \rho_{\min }=-0.16$ e $\AA^{-3}$

\section{Special details}

Geometry. All e.s.d.'s (except the e.s.d. in the dihedral angle between two 1.s. planes) are estimated using the full covariance matrix. The cell e.s.d.'s are taken into account individually in the estimation of e.s.d.'s in distances, angles and torsion angles; correlations between e.s.d.'s in cell parameters are only used when they are defined by crystal symmetry. An approximate (isotropic) treatment of cell e.s.d.'s is used for estimating e.s.d.'s involving 1.s. planes.

Refinement. Refinement of $F^{2}$ against ALL reflections. The weighted $R$-factor $w R$ and goodness of fit $S$ are based on $F^{2}$, conventional $R$-factors $R$ are based on $F$, with $F$ set to zero for negative $F^{2}$. The threshold expression of $F^{2}>\sigma\left(F^{2}\right)$ is used only for calculating $R$-factors $(\mathrm{gt})$ etc. and is not relevant to the choice of reflections for refinement. $R$-factors based on $F^{2}$ are statistically about twice as large as those based on $F$, and $R$-factors based on ALL data will be even larger.

Fractional atomic coordinates and isotropic or equivalent isotropic displacement parameters $\left(\AA^{2}\right)$

\begin{tabular}{|c|c|c|c|c|}
\hline & $x$ & $y$ & $z$ & $U_{\text {iso }} * / U_{\text {eq }}$ \\
\hline Ni1 & 0.5000 & 0.5000 & 0.5000 & $0.03193(8)$ \\
\hline O1 & $0.31671(14)$ & $0.39481(6)$ & $0.69080(12)$ & $0.0479(2)$ \\
\hline $\mathrm{H} 1 \mathrm{O}$ & $0.361(2)$ & $0.3665(12)$ & 0.6227 (19) & $0.082(7)^{*}$ \\
\hline $\mathrm{O} 2$ & $0.55255(14)$ & $0.67355(6)$ & $0.52231(12)$ & $0.0491(2)$ \\
\hline N1 & $0.23048(16)$ & $0.52210(8)$ & $0.85500(15)$ & $0.0444(3)$ \\
\hline $\mathrm{H} 1 \mathrm{~N}$ & $0.221(2)$ & $0.4716(9)$ & 0.8697 (19) & $0.047(5)^{*}$ \\
\hline $\mathrm{N} 2$ & $0.37428(14)$ & $0.47315(8)$ & $0.65657(13)$ & $0.0379(2)$ \\
\hline N3 & $0.47882(13)$ & $0.60817(7)$ & $0.57706(13)$ & $0.0373(2)$ \\
\hline $\mathrm{C} 1$ & $0.11259(16)$ & $0.58122(9)$ & $0.90465(15)$ & $0.0391(3)$ \\
\hline $\mathrm{C} 2$ & $-0.01565(17)$ & $0.60907(10)$ & $0.80574(16)$ & $0.0460(3)$ \\
\hline $\mathrm{C} 3$ & $-0.13125(19)$ & $0.66334(11)$ & 0.86028 (19) & $0.0550(4)$ \\
\hline H3 & -0.2169 & 0.6837 & 0.7955 & $0.066^{*}$ \\
\hline $\mathrm{C} 4$ & $-0.1203(2)$ & $0.68714(11)$ & $1.0086(2)$ & $0.0582(4)$ \\
\hline H4 & -0.1994 & 0.7226 & 1.0441 & $0.070 *$ \\
\hline $\mathrm{C} 5$ & $0.0076(2)$ & $0.65839(11)$ & $1.10458(18)$ & $0.0532(4)$ \\
\hline H5 & 0.0139 & 0.6750 & 1.2047 & $0.064 *$ \\
\hline C6 & $0.12720(17)$ & $0.60536(10)$ & $1.05560(16)$ & $0.0439(3)$ \\
\hline $\mathrm{C} 7$ & $0.2701(2)$ & $0.57673(12)$ & 1.15995 (18) & $0.0615(4)$ \\
\hline H7A & 0.2558 & 0.5965 & 1.2597 & $0.092 *$ \\
\hline H7B & 0.3714 & 0.5985 & 1.1264 & $0.092 *$ \\
\hline $\mathrm{H} 7 \mathrm{C}$ & 0.2744 & 0.5169 & 1.1603 & $0.092 *$ \\
\hline $\mathrm{C} 8$ & $-0.0333(2)$ & $0.58167(14)$ & $0.64311(18)$ & $0.0672(5)$ \\
\hline H8A & 0.0450 & 0.6113 & 0.5874 & $0.101 *$ \\
\hline H8B & -0.1434 & 0.5935 & 0.6015 & $0.101 *$ \\
\hline $\mathrm{H} 8 \mathrm{C}$ & -0.0126 & 0.5228 & 0.6372 & $0.101 *$ \\
\hline C9 & $0.32550(16)$ & $0.53464(9)$ & $0.73794(15)$ & $0.0369(3)$ \\
\hline $\mathrm{C} 10$ & $0.39180(16)$ & $0.61579(9)$ & $0.69449(15)$ & 0.0388 (3) \\
\hline $\mathrm{C} 11$ & $0.3760(2)$ & $0.69658(10)$ & $0.7739(2)$ & $0.0606(4)$ \\
\hline H11A & 0.4560 & 0.7353 & 0.7409 & $0.091 *$ \\
\hline
\end{tabular}


supporting information

\begin{tabular}{lllll} 
H11B & 0.3949 & 0.6880 & 0.8803 & $0.091^{*}$ \\
H11C & 0.2668 & 0.7186 & 0.7518 & $0.091^{*}$ \\
\hline
\end{tabular}

Atomic displacement parameters $\left(\AA^{2}\right)$

\begin{tabular}{lllllll}
\hline & $U^{11}$ & $U^{22}$ & $U^{33}$ & $U^{12}$ & $U^{13}$ & $U^{23}$ \\
\hline Ni1 & $0.02994(11)$ & $0.03035(12)$ & $0.03578(12)$ & $0.00224(8)$ & $0.00434(8)$ & $-0.00006(9)$ \\
O1 & $0.0569(6)$ & $0.0344(5)$ & $0.0547(6)$ & $-0.0031(4)$ & $0.0195(5)$ & $0.0013(5)$ \\
O2 & $0.0568(6)$ & $0.0315(5)$ & $0.0612(6)$ & $-0.0033(4)$ & $0.0193(5)$ & $0.0003(4)$ \\
N1 & $0.0461(7)$ & $0.0403(6)$ & $0.0486(7)$ & $0.0048(5)$ & $0.0165(5)$ & $0.0007(5)$ \\
N2 & $0.0381(6)$ & $0.0334(5)$ & $0.0430(6)$ & $0.0003(4)$ & $0.0084(5)$ & $0.0007(5)$ \\
N3 & $0.0346(5)$ & $0.0328(5)$ & $0.0449(6)$ & $0.0009(4)$ & $0.0057(4)$ & $0.0001(5)$ \\
C1 & $0.0359(6)$ & $0.0413(7)$ & $0.0412(7)$ & $0.0013(5)$ & $0.0099(5)$ & $-0.0026(5)$ \\
C2 & $0.0387(7)$ & $0.0566(9)$ & $0.0431(7)$ & $0.0007(6)$ & $0.0058(6)$ & $-0.0020(7)$ \\
C3 & $0.0413(8)$ & $0.0607(10)$ & $0.0635(10)$ & $0.0099(7)$ & $0.0064(7)$ & $0.0075(8)$ \\
C4 & $0.0522(9)$ & $0.0548(10)$ & $0.0701(11)$ & $0.0102(7)$ & $0.0230(8)$ & $-0.0050(8)$ \\
C5 & $0.0601(10)$ & $0.0541(9)$ & $0.0475(8)$ & $-0.0007(7)$ & $0.0181(7)$ & $-0.0102(7)$ \\
C6 & $0.0450(7)$ & $0.0467(8)$ & $0.0406(7)$ & $-0.0027(6)$ & $0.0068(6)$ & $-0.0020(6)$ \\
C7 & $0.0633(10)$ & $0.0719(12)$ & $0.0477(9)$ & $0.0029(9)$ & $-0.0061(7)$ & $0.0019(8)$ \\
C8 & $0.0576(10)$ & $0.0985(16)$ & $0.0443(8)$ & $0.0030(10)$ & $-0.0039(7)$ & $-0.0096(9)$ \\
C9 & $0.0317(6)$ & $0.0387(7)$ & $0.0406(6)$ & $0.0035(5)$ & $0.0045(5)$ & $-0.0020(6)$ \\
C10 & $0.0364(6)$ & $0.0353(6)$ & $0.0451(7)$ & $0.0020(5)$ & $0.0058(5)$ & $-0.0054(5)$ \\
C11 & $0.0628(10)$ & $0.0439(9)$ & $0.0786(11)$ & $-0.0061(7)$ & $0.0292(8)$ & $-0.0192(8)$ \\
& & & & & & \\
\hline
\end{tabular}

Geometric parameters $\left(A,{ }^{\circ}\right)$

\begin{tabular}{|c|c|c|c|}
\hline $\mathrm{N} i 1-\mathrm{N} 2^{\mathrm{i}}$ & $1.8397(11)$ & $\mathrm{C} 3-\mathrm{H} 3$ & 0.9300 \\
\hline $\mathrm{Ni1}-\mathrm{N} 2$ & $1.8397(11)$ & $\mathrm{C} 4-\mathrm{C} 5$ & $1.375(2)$ \\
\hline $\mathrm{Ni} 1-\mathrm{N} 3^{\mathrm{i}}$ & $1.8779(11)$ & $\mathrm{C} 4-\mathrm{H} 4$ & 0.9300 \\
\hline $\mathrm{Ni1}-\mathrm{N} 3$ & $1.8779(11)$ & $\mathrm{C} 5-\mathrm{C} 6$ & $1.384(2)$ \\
\hline $\mathrm{O} 1-\mathrm{N} 2$ & $1.3817(15)$ & $\mathrm{C} 5-\mathrm{H} 5$ & 0.9300 \\
\hline $\mathrm{O} 1-\mathrm{H} 1 \mathrm{O}$ & $0.858(9)$ & $\mathrm{C} 6-\mathrm{C} 7$ & $1.504(2)$ \\
\hline $\mathrm{O} 2-\mathrm{N} 3$ & $1.3181(14)$ & $\mathrm{C} 7-\mathrm{H} 7 \mathrm{~A}$ & 0.9600 \\
\hline $\mathrm{N} 1-\mathrm{C} 9$ & $1.3575(18)$ & $\mathrm{C} 7-\mathrm{H} 7 \mathrm{~B}$ & 0.9600 \\
\hline $\mathrm{N} 1-\mathrm{C} 1$ & $1.4389(17)$ & $\mathrm{C} 7-\mathrm{H} 7 \mathrm{C}$ & 0.9600 \\
\hline $\mathrm{N} 1-\mathrm{H} 1 \mathrm{~N}$ & $0.824(14)$ & $\mathrm{C} 8-\mathrm{H} 8 \mathrm{~A}$ & 0.9600 \\
\hline $\mathrm{N} 2-\mathrm{C} 9$ & $1.3028(19)$ & $\mathrm{C} 8-\mathrm{H} 8 \mathrm{~B}$ & 0.9600 \\
\hline $\mathrm{N} 3-\mathrm{C} 10$ & $1.3111(17)$ & $\mathrm{C} 8-\mathrm{H} 8 \mathrm{C}$ & 0.9600 \\
\hline $\mathrm{C} 1-\mathrm{C} 2$ & $1.387(2)$ & $\mathrm{C} 9-\mathrm{C} 10$ & $1.470(2)$ \\
\hline $\mathrm{C} 1-\mathrm{C} 6$ & $1.3979(19)$ & $\mathrm{C} 10-\mathrm{C} 11$ & $1.486(2)$ \\
\hline $\mathrm{C} 2-\mathrm{C} 3$ & $1.392(2)$ & C11-H11A & 0.9600 \\
\hline $\mathrm{C} 2-\mathrm{C} 8$ & $1.513(2)$ & C11-H11B & 0.9600 \\
\hline $\mathrm{C} 3-\mathrm{C} 4$ & $1.374(2)$ & $\mathrm{C} 11-\mathrm{H} 11 \mathrm{C}$ & 0.9600 \\
\hline $\mathrm{N} 2 \mathrm{i}-\mathrm{Ni} 1-\mathrm{N} 2$ & 180.0 & $\mathrm{C} 4-\mathrm{C} 5-\mathrm{H} 5$ & 119.2 \\
\hline $\mathrm{N} 2^{\mathrm{i}}-\mathrm{Ni} 1-\mathrm{N} 3^{\mathrm{i}}$ & $82.38(5)$ & $\mathrm{C} 6-\mathrm{C} 5-\mathrm{H} 5$ & 119.2 \\
\hline $\mathrm{N} 2-\mathrm{Ni} 1-\mathrm{N} 3^{\mathrm{i}}$ & $97.62(5)$ & $\mathrm{C} 5-\mathrm{C} 6-\mathrm{C} 1$ & $117.53(14)$ \\
\hline $\mathrm{N} 2 \mathrm{i}-\mathrm{Ni} 1-\mathrm{N} 3$ & $97.62(5)$ & $\mathrm{C} 5-\mathrm{C} 6-\mathrm{C} 7$ & $121.19(14)$ \\
\hline
\end{tabular}




\begin{tabular}{|c|c|c|c|}
\hline $\mathrm{N} 2-\mathrm{Ni} 1-\mathrm{N} 3$ & $82.38(5)$ & $\mathrm{C} 1-\mathrm{C} 6-\mathrm{C} 7$ & $121.26(13)$ \\
\hline $\mathrm{N} 3{ }^{\mathrm{i}}-\mathrm{Ni} 1-\mathrm{N} 3$ & 180.0 & $\mathrm{C} 6-\mathrm{C} 7-\mathrm{H} 7 \mathrm{~A}$ & 109.5 \\
\hline $\mathrm{N} 2-\mathrm{O} 1-\mathrm{H} 1 \mathrm{O}$ & $99.1(15)$ & $\mathrm{C} 6-\mathrm{C} 7-\mathrm{H} 7 \mathrm{~B}$ & 109.5 \\
\hline $\mathrm{C} 9-\mathrm{N} 1-\mathrm{C} 1$ & $124.51(13)$ & $\mathrm{H} 7 \mathrm{~A}-\mathrm{C} 7-\mathrm{H} 7 \mathrm{~B}$ & 109.5 \\
\hline $\mathrm{C} 9-\mathrm{N} 1-\mathrm{H} 1 \mathrm{~N}$ & $109.3(12)$ & $\mathrm{C} 6-\mathrm{C} 7-\mathrm{H} 7 \mathrm{C}$ & 109.5 \\
\hline $\mathrm{C} 1-\mathrm{N} 1-\mathrm{H} 1 \mathrm{~N}$ & $121.9(12)$ & $\mathrm{H} 7 \mathrm{~A}-\mathrm{C} 7-\mathrm{H} 7 \mathrm{C}$ & 109.5 \\
\hline $\mathrm{C} 9-\mathrm{N} 2-\mathrm{O} 1$ & $116.31(12)$ & $\mathrm{H} 7 \mathrm{~B}-\mathrm{C} 7-\mathrm{H} 7 \mathrm{C}$ & 109.5 \\
\hline $\mathrm{C} 9-\mathrm{N} 2-\mathrm{Ni} 1$ & $116.99(11)$ & $\mathrm{C} 2-\mathrm{C} 8-\mathrm{H} 8 \mathrm{~A}$ & 109.5 \\
\hline $\mathrm{O} 1-\mathrm{N} 2-\mathrm{Ni} 1$ & $126.55(9)$ & $\mathrm{C} 2-\mathrm{C} 8-\mathrm{H} 8 \mathrm{~B}$ & 109.5 \\
\hline $\mathrm{C} 10-\mathrm{N} 3-\mathrm{O} 2$ & $120.48(11)$ & $\mathrm{H} 8 \mathrm{~A}-\mathrm{C} 8-\mathrm{H} 8 \mathrm{~B}$ & 109.5 \\
\hline $\mathrm{C} 10-\mathrm{N} 3-\mathrm{Ni1}$ & $116.60(9)$ & $\mathrm{C} 2-\mathrm{C} 8-\mathrm{H} 8 \mathrm{C}$ & 109.5 \\
\hline $\mathrm{O} 2-\mathrm{N} 3-\mathrm{Ni} 1$ & $122.85(8)$ & $\mathrm{H} 8 \mathrm{~A}-\mathrm{C} 8-\mathrm{H} 8 \mathrm{C}$ & 109.5 \\
\hline $\mathrm{C} 2-\mathrm{C} 1-\mathrm{C} 6$ & $122.03(13)$ & $\mathrm{H} 8 \mathrm{~B}-\mathrm{C} 8-\mathrm{H} 8 \mathrm{C}$ & 109.5 \\
\hline $\mathrm{C} 2-\mathrm{C} 1-\mathrm{N} 1$ & $120.00(13)$ & $\mathrm{N} 2-\mathrm{C} 9-\mathrm{N} 1$ & $121.96(14)$ \\
\hline $\mathrm{C} 6-\mathrm{C} 1-\mathrm{N} 1$ & $117.88(13)$ & $\mathrm{N} 2-\mathrm{C} 9-\mathrm{C} 10$ & $113.00(12)$ \\
\hline $\mathrm{C} 1-\mathrm{C} 2-\mathrm{C} 3$ & $118.10(14)$ & $\mathrm{N} 1-\mathrm{C} 9-\mathrm{C} 10$ & $124.96(13)$ \\
\hline $\mathrm{C} 1-\mathrm{C} 2-\mathrm{C} 8$ & $122.03(14)$ & $\mathrm{N} 3-\mathrm{C} 10-\mathrm{C} 9$ & $110.90(12)$ \\
\hline $\mathrm{C} 3-\mathrm{C} 2-\mathrm{C} 8$ & $119.87(15)$ & $\mathrm{N} 3-\mathrm{C} 10-\mathrm{C} 11$ & $122.18(13)$ \\
\hline $\mathrm{C} 4-\mathrm{C} 3-\mathrm{C} 2$ & $120.84(16)$ & $\mathrm{C} 9-\mathrm{C} 10-\mathrm{C} 11$ & $126.81(12)$ \\
\hline $\mathrm{C} 4-\mathrm{C} 3-\mathrm{H} 3$ & 119.6 & $\mathrm{C} 10-\mathrm{C} 11-\mathrm{H} 11 \mathrm{~A}$ & 109.5 \\
\hline $\mathrm{C} 2-\mathrm{C} 3-\mathrm{H} 3$ & 119.6 & $\mathrm{C} 10-\mathrm{C} 11-\mathrm{H} 11 \mathrm{~B}$ & 109.5 \\
\hline $\mathrm{C} 3-\mathrm{C} 4-\mathrm{C} 5$ & $119.93(14)$ & $\mathrm{H} 11 \mathrm{~A}-\mathrm{C} 11-\mathrm{H} 11 \mathrm{~B}$ & 109.5 \\
\hline $\mathrm{C} 3-\mathrm{C} 4-\mathrm{H} 4$ & 120.0 & $\mathrm{C} 10-\mathrm{C} 11-\mathrm{H} 11 \mathrm{C}$ & 109.5 \\
\hline $\mathrm{C} 5-\mathrm{C} 4-\mathrm{H} 4$ & 120.0 & $\mathrm{H} 11 \mathrm{~A}-\mathrm{C} 11-\mathrm{H} 11 \mathrm{C}$ & 109.5 \\
\hline $\mathrm{C} 4-\mathrm{C} 5-\mathrm{C} 6$ & $121.55(15)$ & $\mathrm{H} 11 \mathrm{~B}-\mathrm{C} 11-\mathrm{H} 11 \mathrm{C}$ & 109.5 \\
\hline $\mathrm{N} 3{ }^{\mathrm{i}}-\mathrm{Ni} 1-\mathrm{N} 2-\mathrm{C} 9$ & $-177.23(10)$ & $\mathrm{C} 4-\mathrm{C} 5-\mathrm{C} 6-\mathrm{C} 7$ & $177.73(16)$ \\
\hline $\mathrm{N} 3-\mathrm{Ni} 1-\mathrm{N} 2-\mathrm{C} 9$ & $2.77(10)$ & $\mathrm{C} 2-\mathrm{C} 1-\mathrm{C} 6-\mathrm{C} 5$ & $0.3(2)$ \\
\hline $\mathrm{N} 3{ }^{\mathrm{i}}-\mathrm{Ni} 1-\mathrm{N} 2-\mathrm{O} 1$ & $-1.92(12)$ & $\mathrm{N} 1-\mathrm{C} 1-\mathrm{C} 6-\mathrm{C} 5$ & $-176.24(14)$ \\
\hline $\mathrm{N} 3-\mathrm{Ni} 1-\mathrm{N} 2-\mathrm{O} 1$ & $178.08(12)$ & $\mathrm{C} 2-\mathrm{C} 1-\mathrm{C} 6-\mathrm{C} 7$ & $-178.00(15)$ \\
\hline $\mathrm{N} 2{ }^{\mathrm{i}}-\mathrm{Ni} 1-\mathrm{N} 3-\mathrm{C} 10$ & $179.36(10)$ & $\mathrm{N} 1-\mathrm{C} 1-\mathrm{C} 6-\mathrm{C} 7$ & $5.4(2)$ \\
\hline $\mathrm{N} 2-\mathrm{Ni1}-\mathrm{N} 3-\mathrm{C} 10$ & $-0.64(10)$ & $\mathrm{O} 1-\mathrm{N} 2-\mathrm{C} 9-\mathrm{N} 1$ & $3.3(2)$ \\
\hline $\mathrm{N} 2{ }^{\mathrm{i}}-\mathrm{Ni} 1-\mathrm{N} 3-\mathrm{O} 2$ & $-3.65(12)$ & $\mathrm{Ni} 1-\mathrm{N} 2-\mathrm{C} 9-\mathrm{N} 1$ & $179.15(10)$ \\
\hline $\mathrm{N} 2-\mathrm{Ni} 1-\mathrm{N} 3-\mathrm{O} 2$ & $176.35(12)$ & $\mathrm{O} 1-\mathrm{N} 2-\mathrm{C} 9-\mathrm{C} 10$ & $-179.93(11)$ \\
\hline $\mathrm{C} 9-\mathrm{N} 1-\mathrm{C} 1-\mathrm{C} 2$ & $57.9(2)$ & $\mathrm{Ni1}-\mathrm{N} 2-\mathrm{C} 9-\mathrm{C} 10$ & $-4.13(15)$ \\
\hline $\mathrm{C} 9-\mathrm{N} 1-\mathrm{C} 1-\mathrm{C} 6$ & $-125.45(16)$ & $\mathrm{C} 1-\mathrm{N} 1-\mathrm{C} 9-\mathrm{N} 2$ & $-150.05(14)$ \\
\hline $\mathrm{C} 6-\mathrm{C} 1-\mathrm{C} 2-\mathrm{C} 3$ & $0.6(2)$ & $\mathrm{C} 1-\mathrm{N} 1-\mathrm{C} 9-\mathrm{C} 10$ & $33.6(2)$ \\
\hline $\mathrm{N} 1-\mathrm{C} 1-\mathrm{C} 2-\mathrm{C} 3$ & $177.13(14)$ & $\mathrm{O} 2-\mathrm{N} 3-\mathrm{C} 10-\mathrm{C} 9$ & $-178.35(11)$ \\
\hline $\mathrm{C} 6-\mathrm{C} 1-\mathrm{C} 2-\mathrm{C} 8$ & $-178.83(15)$ & $\mathrm{Ni} 1-\mathrm{N} 3-\mathrm{C} 10-\mathrm{C} 9$ & $-1.29(15)$ \\
\hline $\mathrm{N} 1-\mathrm{C} 1-\mathrm{C} 2-\mathrm{C} 8$ & $-2.3(2)$ & $\mathrm{O} 2-\mathrm{N} 3-\mathrm{C} 10-\mathrm{C} 11$ & $-1.9(2)$ \\
\hline $\mathrm{C} 1-\mathrm{C} 2-\mathrm{C} 3-\mathrm{C} 4$ & $-1.4(2)$ & $\mathrm{Ni1}-\mathrm{N} 3-\mathrm{C} 10-\mathrm{C} 11$ & $175.16(12)$ \\
\hline $\mathrm{C} 8-\mathrm{C} 2-\mathrm{C} 3-\mathrm{C} 4$ & $178.11(16)$ & $\mathrm{N} 2-\mathrm{C} 9-\mathrm{C} 10-\mathrm{N} 3$ & $3.42(17)$ \\
\hline $\mathrm{C} 2-\mathrm{C} 3-\mathrm{C} 4-\mathrm{C} 5$ & $1.1(3)$ & $\mathrm{N} 1-\mathrm{C} 9-\mathrm{C} 10-\mathrm{N} 3$ & $-179.97(13)$ \\
\hline $\mathrm{C} 3-\mathrm{C} 4-\mathrm{C} 5-\mathrm{C} 6$ & $-0.1(3)$ & $\mathrm{N} 2-\mathrm{C} 9-\mathrm{C} 10-\mathrm{C} 11$ & $-172.82(14)$ \\
\hline $\mathrm{C} 4-\mathrm{C} 5-\mathrm{C} 6-\mathrm{C} 1$ & $-0.6(2)$ & $\mathrm{N} 1-\mathrm{C} 9-\mathrm{C} 10-\mathrm{C} 11$ & $3.8(2)$ \\
\hline
\end{tabular}

Symmetry code: (i) $-x+1,-y+1,-z+1$. 


\section{supporting information}

Hydrogen-bond geometry $\left(A,{ }^{\circ}\right)$

\begin{tabular}{lllll}
\hline$D-\mathrm{H} \cdots A$ & $D-\mathrm{H}$ & $\mathrm{H} \cdots A$ & $D \cdots A$ & $D-\mathrm{H} \cdots A$ \\
\hline $\mathrm{O} 1-\mathrm{H} 1 O \cdots \mathrm{O} 2^{\mathrm{i}}$ & $0.86(1)$ & $1.65(1)$ & $2.4972(14)$ & $171(2)$ \\
$\mathrm{N} 1-\mathrm{H} 1 N \cdots \mathrm{O} 1$ & $0.82(1)$ & $2.20(2)$ & $2.6361(16)$ & $113(1)$ \\
\hline
\end{tabular}

Symmetry code: (i) $-x+1,-y+1,-z+1$. 